\title{
Processing costed inpatient episodes to cost weights for inlier equivalent separations: the ends should fit the means
}

\author{
C W Aisbett ${ }^{1,2}$ \\ From 26th Patient Classification Systems International (PCSI) Working Conference \\ Munich, Germany. 15-18 September 2010
}

\section{Introduction}

A principal reason for investing in case mix as a tool for hospital administration is that it allows the value of production embodied in an episode of care to be different from the cost of that episode.

Useful case-mix evaluation retains a statistical nexus between value and cost of production. The value placed on a hospital system's output should fit with the aggregate cost of the episodes used to make that evaluation. This should apply at the case-type level.

Case-mix funding and evaluation are seldom based on average cost of episodes within the same DRG. They are supplemented with risk sharing between funder and provider, and with compensatory factors for structural differences.

Risk and structure are modelled. A standard value (cost weight) is assigned to each DRG. The value is related to the system-wide average cost of a LOS inlier (corrected for structural factors) in that DRG. The notion is that except for a constant multiplicative factor, the cost weight for a DRG is the expected cost of a LOS inlier episode in that DRG across the hospital system (after removing structural effects).

Once risk and structure are accounted for, the fit between DRG level aggregate cost and value is harder to maintain. Almost invariably, the relative values assigned to different DRGs are distorted.

The contention of this article is that if a collection of activity-based costing data has provided costs by DRG for a number of hospitals, then the result of applying any inlier equivalent cost weights generated from the data should return the system-wide cost of each DRG.

${ }^{1}$ LaetaPty Ltd, Randwick, New South Wales, Australia

Full list of author information is available at the end of the article
The contribution of this article is an example and methodology to achieve this when the modelling of inlier equivalent separations includes policy, as well as data determined structure and parameters.

\section{Methods}

The NSW Department of Health mandates an annual activity-based costing of each of its larger acute hospitals. The focus of this article is inpatient care, for which the costing methodology is standardised and supported by a state-wide costing system, PCM, provided by Power HealthSolutions.

Inlier data from four years and 52 hospitals were used to obtain estimates of the DRG upper and lower trim points and per-diem payment for each type of outlier. Short stay transfers $(\mathrm{LOS}=1)$ were also treated as outliers and assigned DRG-specific costs. These parameters were fixed throughout the iterative process that follows.

Firstly, hospital by AR-DRG data cells were processed to obtain cost and utilisation statistics. The latter included outlier days of various types that were broken down by public/private and indigenous status. These summaries were the "units" of the analysis.

Iteration starts with subjecting the "units" to automatic plausibility checks and statistical trimming based on current values of cost per inlier equivalent. Thus hospital by DRG data are swapped in and out of calculations.

Next, case-mix methods (e.g., indirect standardisation) are used to calculate multiplicative hospital effects and, if extreme, the whole of the hospital's data are removed from the "unit" cost data.

The adjusted data are then passed through an aggregate function providing adjusted cost per equivalent 
separation. When divided by the cost base, these are "current value" cost weights.

The current values are compared with the previous iteration and if "different" are passed to the next iteration. Otherwise, the current values are returned as the cost weights.

\section{Results}

The cost weights converged after less than 10 iterations. The values were very similar to the weights derived using the regression-based method used in previous years.

A significant gain from the iterative approach was the relative simplicity of the algorithm, which allowed automated trimming and reselection of suspect data based on current values of the cost weight (at each iteration), rather than just trimming based on initial estimates.

An important aside here is that the iterations showed the effects of data trimming on the final estimates and, hence, uncovered AR-DRG with weights that were highly sensitive to the data trimming algorithm used. In particular, A12Z INS NEUROSTIMULATOR DEV had values ranging from as low as $\$ 7000$ to as high as $\$ 22,000$ before settling at $\$ 17,000$. Review of the cases indicated all cases in one hospital were (low) cost outliers.

\section{Conclusions}

Once "external "parameters such as the various perdiem rates and multiplicative adjustments for public/private and indigenous status are settled on, the iterative process is not significantly more difficult to program, or execute, than the one-step approach upon which it was based. Further, it reduces the dependence on initial estimates used in data cleaning, provides sensitivity information on cost weights, and satisfies the requirement that the recorded system-wide cost per DRG be reconciled with the value assigned to activity in that AR-DRG.

\section{Author details}

${ }^{1}$ LaetaPty Ltd, Randwick, New South Wales, Australia. ${ }^{2}$ Department of Health and Children, Dublin, Ireland.

Published: 6 October 2010

doi:10.1186/1472-6963-10-S2-A15

Cite this article as: Aisbett: Processing costed inpatient episodes to cost weights for inlier equivalent separations: the ends should fit the means. BMC Health Services Research 2010 10(Suppl 2):A15.
Submit your next manuscript to BioMed Central and take full advantage of:

- Convenient online submission

- Thorough peer review

- No space constraints or color figure charges

- Immediate publication on acceptance

- Inclusion in PubMed, CAS, Scopus and Google Scholar

- Research which is freely available for redistribution

Submit your manuscript at www.biomedcentral.com/submit 